6. G. Lippmann, La photographie des couleurs, Revue générale des sciences pures et appliquées, 1891, pp. 161-172.

7. E. Le Coarer and P. Benech, French patent 04/52992, 2004.

8. Avago Technologies, Agilent HSCH-9161 GaAs detector diode sensitivity measurements, product note no. 12, 1999.

9. N. Roddis, D. Kettle, F. Winder, B. Aja, E. Artal, M.L. de la Fuente, J.P. Pascual, A. Mediavilla, L. Pradell, and P. de Paco, Differential radiometer at $30 \mathrm{GHz}$ for the Planck emission, In: Third ESA workshop on millimetre wave technology and applications: Circuits, systems, and measurement techniques, Millilab, Espoo, Finland, 21-23 May, 2003, pp. 81-86.

10. E. Artal, B. Aja, M.L. de la Fuente, N. Roddis, D. Kettle, F. Winder, L. Pradell, and P. de Paco, Radiometers at 30 and $44 \mathrm{GHz}$ for the Plank emission, In: Microwave technology and techniques workshop, European Space Agency-CNES, Nordwijk, Nederlands, October 2002, pp. 41-48.

(C) 2007 Wiley Periodicals, Inc.

\section{A NEW DERIVATION FOR THE COEFFICIENTS OF THE SCATTERING DYADIC GREEN'S FUNCTION IN SPHERICALLY LAYERED MEDIA}

Yonghoon Kim, Heeduck Chae, and Sangwook Nam

132-308 Seoul National University, San 56-1, Sillim-dong,

Gwanak-gu, Seoul, 151-742, Republic of Korea

Received 2 October 2006

ABSTRACT: The dyadic Green's function in spherically layered media is considered by assuming that source and field points can be located anywhere. After manipulations from the expression of the dyadic Green's function based on the reflections and transmissions of the scalar waves, the dyadic Green's function in terms of the spherical vector wave functions is newly constructed and also the coefficients of the scattering dyadic Green's function are derived in a simple form. () 2007 Wiley Periodicals, Inc. Microwave Opt Technol Lett 49: 1142-1143, 2007; Published online in Wiley InterScience (www.interscience.wiley. com). DOI 10.1002/mop.22374

Key words: electromagnetic waves; Dyadic Green's function; scattering coefficient; spherically layered media; vector wave function

\section{INTRODUCTION}

The dyadic Green's function (DGF) has been an interesting topic in electromagnetic theory. Although most problems can be solved without the use of DGFs, the symbolic simplicity with which they could be used to express relationships makes the formulations of many problems simpler and more compact. The DGFs for simple spherical geometries were introduced earlier [1], and a general expression of DGF for a spherically arbitrary layered medium was constructed [2]. In both works, the DGFs in terms of the spherical vector wave functions (VWFs) were first expressed in each layer using the method of scattering superposition, and then the unknown coefficients within them were solved based on the coupling

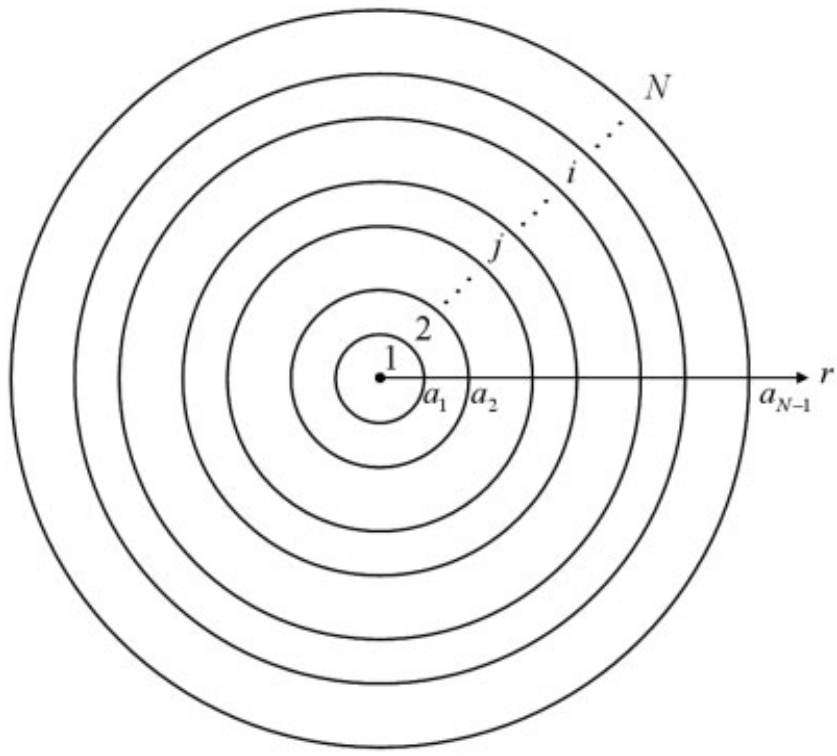

Figure 1 Geometry of spherical of $N$-layered medium

recurrence matrix equations by imposing boundary conditions at all interfaces. In this article, starting from the DGF formulated by Chew [3], which is based on the reflections and transmissions of the scalar waves characterized by Debye potentials, a simple and direct way of deriving the coefficients of the scattering DGF when expressed in terms of spherical VWFs is presented. The timedependent $\exp (-i \omega t)$ is assumed for the field quantities throughout the paper.

\section{GENERAL EXPRESSION OF DGF}

The spherical concentric $N$-layered medium under consideration is shown in Figure 1. The complex permittivity and permeability of $i$ th layer are described by $\varepsilon_{i}$ and $\mu_{i}$, respectively. The DGF of electric type for a spherically layered medium is given in Ref. 3 (Eq. 7.4.32).

$$
\overline{\mathbf{G}}_{e}^{(i j)}\left(\mathbf{r}, \mathbf{r}^{\prime}\right)=i k_{j} \sum_{n=0}^{\infty} \frac{1}{n(n+1)}\left[\overline{\mathbf{m}}_{n}\left(\mathbf{r}, \mathbf{r}^{\prime}\right)+\overline{\mathbf{n}}_{n}\left(\mathbf{r}, \mathbf{r}^{\prime}\right)\right] \quad\left(\mathbf{r} \neq \mathbf{r}^{\prime}\right)
$$

where $k_{j}=k_{0} \sqrt{\varepsilon_{j} \mu_{j}}$, and it is assumed that the source point $\mathbf{r}^{\prime}$ is in the layer $j$ and the field point $\mathbf{r}$ is in the layer $i(i, j=1,2, \ldots$, $N) . \overline{\mathbf{m}}_{n}$ and $\overline{\mathbf{n}}_{n}$ in Eq. (1) are the quantities that are proportional to the Debye potentials and defined by [3]:

$$
\begin{gathered}
\overline{\mathbf{m}}_{n}\left(\mathbf{r}, \mathbf{r}^{\prime}\right)=(\nabla \times \mathbf{r})\left(\nabla^{\prime} \times \mathbf{r}^{\prime}\right) F_{n}^{\mathrm{TE}}\left(r, r^{\prime}\right) A_{n}\left(\theta, \phi ; \theta^{\prime}, \phi^{\prime}\right) \\
\overline{\mathbf{n}}_{n}\left(\mathbf{r}, \mathbf{r}^{\prime}\right)=\left(\frac{\nabla \times \nabla \times \mathbf{r}}{-i \omega \varepsilon_{i}}\right)\left(\frac{\nabla^{\prime} \times \nabla^{\prime} \times \mathbf{r}^{\prime}}{i \omega \mu_{j}}\right) F_{n}^{\mathrm{TM}}\left(r, r^{\prime}\right) A_{n}\left(\theta, \phi ; \theta^{\prime}, \phi^{\prime}\right)
\end{gathered}
$$

where 


$$
A_{n}\left(\theta, \phi ; \theta^{\prime}, \phi^{\prime}\right)=\sum_{m=-n}^{n} Y_{n m}(\theta, \phi) Y_{n,-m}\left(\theta^{\prime}, \phi^{\prime}\right)
$$

$\tilde{R}, \tilde{T}$, and $\tilde{M}_{j}=\left(1-\tilde{R}_{j, j+1} \tilde{R}_{j, j-1}\right)^{-1}$ in Eq. (4) are generalized reflection/transmission coefficients for either TE or TM waves and a factor accounting for multiple reflections in the layer $j$, respectively, and $Y_{n m}$ in Eq. (5) is a spherical harmonic function [3]. Using that $\tilde{M}_{j}=1+\left(\tilde{M}_{j}-1\right)=1+\tilde{R}_{j, j+1} \tilde{R}_{j, j-1} \tilde{M}_{j}, F_{n}$ can be separated into two terms, an unbounded (direct) term a scattering one:

$$
F_{n}\left(r, r^{\prime}\right)=F_{n d}\left(r, r^{\prime}\right) \delta_{i j}+F_{n s}\left(r, r^{\prime}\right)
$$

where $\delta_{i j}$ is the Kronecker delta function and

$$
\begin{gathered}
F_{n d}\left(r, r^{\prime}\right)= \begin{cases}h_{n}^{(1)}\left(k_{j} r\right) j_{n}\left(k_{j} r^{\prime}\right), & r>r^{\prime} \\
j_{n}\left(k_{j} r\right) h_{n}^{(1)}\left(k_{j} r^{\prime}\right), & r<r^{\prime}\end{cases} \\
F_{n s}\left(r, r^{\prime}\right)=A_{n} h_{n}^{(1)}\left(k_{i} r\right) j_{n}\left(k_{j} r^{\prime}\right)+B_{n} h_{n}^{(1)}\left(k_{i} r\right) h_{n}^{(1)}\left(k_{j} r^{\prime}\right) \\
+C_{n} j_{n}\left(k_{i} r\right) j_{n}\left(k_{j} r^{\prime}\right)+D_{n} j_{n}\left(k_{i} r\right) h_{n}^{(1)}\left(k_{j} r^{\prime}\right)
\end{gathered}
$$

$A_{n}$ to $D_{n}$ in Eq. (8) are the coefficients of the scattering DGF for either TE and TM waves and can be expressed in terms of $\tilde{R}, \tilde{T}$, and $\tilde{M}$, which will be explained later in detail. By substituting (2) and (3) back into (1), after some manipulations, $\overline{\mathbf{G}}_{e}^{(i j)}\left(\mathbf{r}, \mathbf{r}^{\prime}\right)$ also can be separated into two terms and constructed in terms of the spherical VWFs, $\mathbf{M}$ and $\mathbf{N}$ [1]:

$$
\begin{aligned}
& \overline{\mathbf{G}}_{e}^{(i j)}\left(\mathbf{r}, \mathbf{r}^{\prime}\right)=\overline{\mathbf{G}}_{e 0}\left(\mathbf{r}, \mathbf{r}^{\prime}\right) \delta_{i j}+\overline{\mathbf{G}}_{e s}^{(i j)}\left(\mathbf{r}, \mathbf{r}^{\prime}\right) \\
& \overline{\mathbf{G}}_{e 0}\left(\mathbf{r}, \mathbf{r}^{\prime}\right)=\frac{i k_{j}}{4 \pi} \sum_{n=1}^{\infty} \sum_{m=0}^{n}\left(2-\delta_{m 0}\right) \frac{2 n+1}{n(n+1)} \frac{(n-m) !}{(n+m) !}
\end{aligned}
$$

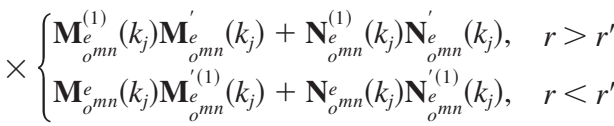

$$
\begin{aligned}
& \overline{\mathbf{G}}_{e s}^{(i j)}\left(\mathbf{r}, \mathbf{r}^{\prime}\right)=\frac{i k_{j}}{4 \pi} \sum_{n=1}^{\infty} \sum_{m=0}^{n}\left(2-\delta_{m 0}\right) \frac{2 n+1}{n(n+1)} \frac{(n-m) !}{(n+m) !} \\
& \times\left[( 1 - \delta _ { i 1 } ) \mathbf { M } _ { o ^ { m } m } ^ { ( 1 ) } ( k _ { i } ) \left\{\left(1-\delta_{j N}\right) A_{n}^{\mathrm{TE}} \mathbf{M}_{o_{o m n}^{\prime}}^{\prime}\left(k_{j}\right)\right.\right. \\
& \left.+\left(1-\delta_{j 1}\right) B_{n}^{\mathrm{TE}} \mathbf{M}_{o^{m n}}^{{ }^{\prime}(1)}\left(k_{j}\right)\right\}+\left(1-\delta_{i N}\right) \mathbf{M}_{o^{m n}}{ }^{m}\left(k_{i}\right)\{(1 \\
& \left.\left.-\delta_{j N}\right) C_{n}^{\mathrm{TE}} \mathbf{M}_{o^{m n}}^{\prime}\left(k_{j}\right)+\left(1-\delta_{j 1}\right) D_{n}^{\mathrm{TE}} \mathbf{M}_{o^{m n}}^{\prime(1)}\left(k_{j 1}\right)\right\} \\
& +\left(1-\delta_{i 1}\right) \mathbf{N}_{e^{m n}}^{(1)}\left(k_{i}\right)\left\{\left(1-\delta_{j N}\right) A_{n}^{\mathrm{TM}} \mathbf{N}_{o^{m n}}^{\prime}\left(k_{j}\right)\right. \\
& \left.+\left(1-\delta_{j 1}\right) B_{n}^{\mathrm{TM}} \mathbf{N}_{o^{m n}}{ }^{\prime(1)}\left(k_{j}\right)\right\} K^{\mathrm{TM}}+\left(1-\delta_{i N}\right) \mathbf{N}_{o}{ }^{e}{ }^{m n}\left(k_{i}\right) \\
& \left.\times\left\{\left(1-\delta_{j N}\right) C_{n}^{\mathrm{TM}} \mathbf{N}_{o_{o}{ }^{\prime} n}^{\prime}\left(k_{j}\right)+\left(1-\delta_{j 1}\right) D_{n}^{\mathrm{TM}} \mathbf{N}_{o_{o}}^{\prime(1)}\left(k_{j}\right)\right\} K^{\mathrm{TM}}\right]
\end{aligned}
$$

where $K^{\mathrm{TM}}=\sqrt{\left(\varepsilon_{j} \mu_{i}\right) /\left(\varepsilon_{i} \mu_{j}\right)}$ is a constant for TM modes and $\overline{\mathbf{G}}_{e 0}\left(\mathbf{r}, \mathbf{r}^{\prime}\right)$ represents the contribution of the direct waves while $\overline{\mathbf{G}}_{e s}^{(i j)}\left(\mathbf{r}, \mathbf{r}^{\prime}\right)$ describes that of the multiple reflections and transmissions of waves in the presence of a layered medium.
TABLE 1 Coefficients of the Scattering DGF Assuming That the Source Point is in Region $j$ and the Field Point is in Region $i$

\begin{tabular}{llll}
\hline & \multicolumn{1}{c}{$i=j$} & \multicolumn{1}{c}{$i>j$} & \multicolumn{1}{c}{$i<j$} \\
\hline$A_{\mathrm{n}}$ & $\tilde{R}_{j, j-1} \tilde{M}_{j} \tilde{R}_{j, j+1}$ & $\tilde{M}_{i} \tilde{T}_{j i} \tilde{M}_{j}$ & $\tilde{R}_{i, i-1} \tilde{M}_{i} \tilde{T}_{j i} \tilde{M}_{j} \tilde{R}_{j, j+1}$ \\
$B_{\mathrm{n}}$ & $\tilde{M}_{j} \tilde{R}_{j, j-1}$ & $\tilde{M}_{i} \tilde{T}_{j i} \tilde{M}_{j} \tilde{R}_{j, j-1}$ & $\tilde{R}_{i, i-1} \tilde{M}_{i} \tilde{T}_{j i} \tilde{M}_{j}$ \\
$C_{\mathrm{n}}$ & $\tilde{M}_{j} \tilde{R}_{j, j+1}$ & $\tilde{R}_{i, i+1} \tilde{M}_{i} \tilde{T}_{j i} \tilde{M}_{j}$ & $\tilde{M}_{i} \tilde{T}_{j i} \tilde{M}_{j} \tilde{R}_{j, j+1}$ \\
$D_{\mathrm{n}}$ & $\tilde{R}_{j, j+1} \tilde{M}_{j} \tilde{R}_{j, j-1}$ & $\tilde{R}_{i, i+1} \tilde{M}_{i} \tilde{T}_{j i} \tilde{M}_{j} \tilde{R}_{j, j-1}$ & $\tilde{M}_{i} \tilde{T}_{j i} \tilde{M}_{j}$ \\
\hline
\end{tabular}

\section{COEFFICIENTS OF SCATTERING DGF}

Since the unbounded DGF $\overline{\mathbf{G}}_{e 0}\left(\mathbf{r}, \mathbf{r}^{\prime}\right)$ is usually evaluated analytically from the spatial representation $\overline{\mathbf{G}}_{e 0}\left(\mathbf{r}, \mathbf{r}^{\prime}\right)=(\overline{\mathbf{I}}$ $\left.+\nabla \nabla / k^{2}\right) G_{0}\left(\mathbf{r}, \mathbf{r}^{\prime}\right)$, where $G_{0}\left(\mathbf{r}, \mathbf{r}^{\prime}\right)$ is the scalar Green's function, only the scattering DGF and its coefficients are considered here. In deriving Eq. (8), four coefficients can be expressed as shown in Table 1 , and these are easily calculated using the single-interface reflection/transmission coefficients. Physically, the coefficients $A_{n}$ and $B_{n}$ represent the outgoing waves while $C_{n}$ and $D_{n}$ represent the standing waves in the field region $i$. In addition, $A_{n}$ and $C_{n}$ represent the waves whose initial reflections or transmissions are occurred at the outer interface while $B_{n}$ and $D_{n}$ represent the waves whose initial reflections or transmissions are occurred at the inner interface of the source region $j$.

\section{CONCLUSION}

The dyadic Green's function has been newly constructed in terms of the spherical vector wave functions based on the reflections and transmissions of the scalar waves characterized by Debye potentials. The coefficients of the scattering dyadic Green's function also have been derived in this process, which is compact, intuitively derivable and suitable to elucidate the physics better.

\section{ACKNOWLEDGMENT}

This work was supported in part by MIC and IITA of Korea through IT Leading R\&D Support Project.

\section{REFERENCES}

1. C.T. Tai, Dyadic Green functions in electromagnetic theory, IEEE Press, New York, 1994.

2. L.W. Li, P.S. Kooi, M.S. Leong, and T.S. Yeo, Electromagnetic dyadic Green's function in spherically multilayered media, IEEE Trans Microwave Theory Technol MTT-42 (1994), 2302-2310.

3. W.C. Chew, Waves and fields in inhomogeneous media, Van Nostrand Reinhold, New York, 1990.

C) 2007 Wiley Periodicals, Inc. 